\title{
Constructing Hybrid Protein Zymogens through Protective Dendritic Assembly
}

\author{
David Y.W. Ng, Matthias Arzt, Yuzhou Wu, Seah Ling Kuan, Markus Lamla and Tanja Weil*
}

\begin{abstract}
Modulation of protein uptake and activity in response to physiological changes forms an integral part of smart protein therapeutics. We describe herein the self-assembly of a $\mathrm{pH}$ responsive dendrimer shell onto the periphery of active enzymes (trypsin, papain, DNase I) as a supramolecular protecting group to form a hybrid dendrimer-enzyme complex. The attachment based on boronic acid/salicyl hydroxamate interaction allows the macromolecular assembly to respond to changes in $\mathrm{pH}$ between 5.0 and 7.4 in a highly reversible fashion. Catalytic activity is efficiently blocked in the presence of the dendrimer shell but is quantitatively restored upon shell degradation in acidic conditions. Unlike the native proteases, the hybrid constructs are shown to be efficiently uptaken into A549 cells and co-localized in the acidic compartments. The programmed intracellular release of the proteases induced cytotoxicity, thereby uncovering a new avenue for precision biotherapeutics.
\end{abstract}

The application of stimulus responsive protective groups offering precise control of protein activity is an appealing approach in the preparation of protein based drugs with potentially high therapeutic index. ${ }^{[1]}$ In this aspect, Nature introduces peptidic protecting groups to proteases (trypsin, pepsin, caspases) resulting in the formation of zymogens, facilitating efficient intra/extracellular transportation and preventing unintended cellular degradation. ${ }^{[2]}$ Inspired by this transcendental efficacy, the introduction of synthetic macromolecular protecting groups provides a contemporary chemical perspective by masking the function of an enzyme through steric bulk prior to exposure to a designated stimulus.

Proteases play an intricate and central role in cancer progression and metastasis, regulating processes such as proliferation, apoptosis, differentiation and evasion of the immune system. ${ }^{[3]}$ Delivery of proteases is important in the elucidation of their function as well as the development of new protein therapeutics. As such, the design of a synthetic strategy incorporating a responsive "assemble and release" system mimicking the biosynthesis of zymogens represents an ideal model. Importantly, the innate proteolytic activity of proteases precludes them from most biological delivery systems (i.e. cell penetrating peptides, fusion proteins).

[*] D.Y.W. Ng, M. Arzt, Y. Wu, Dr. S.L. Kuan, M. Lamla, Prof. Dr. T. Weil.

Institute of Organic Chemistry III

Ulm University

Albert-Einstein-Allee 11, 89081 Ulm, Germany

E-mail: tanja.weil@uni-ulm.de

D.Y.W. Ng, Prof. Dr. T. Weil.

Max Planck Institute for Polymer Research

Ackermannweg 10, Mainz, 55128 (Germany)

[**] This work is supported by German research foundation (DFG) within the SFB 625, the BMBF project Biotechnologie 2020+ and the ERC Synergy Grant 319130BioQ. SLK is grateful to the Alexander von Humboldt Foundation for providing a research fellowship.

Supporting information for this article is available on the WWW under http://www.angewandte.org or from the author.
Modification of active proteases to include stimulus responsive moieties has been challenged by protein denaturation, postmodification inactivation as well as chemoselectivity which often necessitates the use of mild bioorthogonal approaches. ${ }^{[4]}$ Conventionally, site-specific modification through single residue mutation had been developed to incorporate thermoresponsive steric handles near the catalytic site acting as a temperature dependent switch. ${ }^{[5-7]}$ This concept was subsequently evolved into chemical strategies towards the development of protein-polymer conjugates assembled using in situ atom transfer radical polymerisation. ${ }^{[8,9]}$ However, site specific mutation of different classes of enzymes are often challenging with their highly diversed activity and stability whereas metal catalysed reactions on proteins are plagued by residual adsorption of metal contaminants. As a result, the application of these enzymatic constructs is still highly confined and under-developed.

In this aspect, macromolecular protecting groups involving metal-free chemistry utilizing physiological conditions for protection/deprotection of enzyme catalytic sites offer a distinct advantage over existing systems. Inspired by recent results on engineered aptamers ${ }^{[10]}$ as macromolecular protecting groups, we report herein the use of a stimulus responsive dendritic core shell system that self assembles into a supramolecular dendrimer-enzyme complex in a facile and bioorthogonal manner. Small defined dendrimer segments (dendrons) provide high volume to molecular weight ratio due to their branched like structure and thus serves as an ideal candidate as a sterical protecting group. ${ }^{[11]}$ In addition, the dendrons confer additional pharmacologically attractive properties (increased uptake, potency) $^{[12]}$ depending on their surface functionalities. ${ }^{[13]}$ These dendrons are self-assembled onto the enzyme utilizing an acid labile boronic acid/salicyl hydroxamate based ligation method, resulting in a $\mathrm{pH}$ responsive dendrimerenzyme hybrid construct functioning as a synthetic zymogen (Scheme 1a). We have chosen catalytically unique enzymes (trypsin, papain, DNase I), each representing an enzyme class with high therapeutic significance to demonstrate the broad applicability of this strategy. ${ }^{[3]}$

The synthesis of the steric dendrimer protective group builds upon the bi-functional second generation poly(amido)amine dendron bearing a single azido group as well as four primary amine groups $\left(\mathrm{N}_{3}-\mathrm{G} 2\right.$ PAMAM, Scheme 1b) that has been described previously. ${ }^{[14]}$ The azido core group of the dendron was derivatized into a salicyl hydroxamate moiety via copper catalysed azide-alkyne cycloaddition. Salicyl hydroxamates have been previously characterized to "click" with aryl boronic acids to form a stable boronate complex. ${ }^{[15]}$ The kinetics and orthogonality of this ultrafast complexation were studied and had been utilized to perform efficient peptide ligation. ${ }^{[16]}$ To construct the dendron core group, 4pentynoic acid was activated by $N$-hydroxysuccinimide and coupled onto 4-aminosalicyclic acid to afford 3. Condensation of the ethynyl salicyclic acid $\mathbf{3}$ with trityloxyamine in excess afforded $\mathbf{4}$ in moderate yield. The resulting protected ethynyl hydroxamic acid underwent copper catalysed cycloaddition with boc-protected azido PAMAM generation 2 dendron with high yield. Subsequent deprotection using trifluoroacetic acid in the presence of triisopropylsilane as a radical scavenger afforded the final product 5 as a trifluoroacetate salt. 
The native enzymes trypsin, papain and DNase I were functionalized via condensation on the surface lysine residues with an excess of pre-activated 4-carboxyl boronic acid (Scheme 1a). The catalytic domains of the proteases primarily do not involve lysine residues, making them an attractive modification target. ${ }^{[17-19]}$ The modified proteins were purified using ultrafiltration followed by MALDI-TOF MS characterization (Figure S1 in Supporting Information). Trypsin, papain and DNase I were functionalized with an average of 10,10 and 4 boronic acid residues respectively due to their differences in the number of solvent accessible lysines. The degree of functionalization for trypsin and papain is $90 \%$ whereas only $50 \%$ of the surface amines were modified for DNase I. These modified proteins were subsequently assayed for their catalytic activity which showed a conservation of $>80 \%$ compared to their native counterparts (Figure S2 in Supporting Information).

The construction of the dendritic assembly with dendron $\mathbf{5}$ was monitored using a fluorescence assay based on Alizarin Red S. Alizarin Red S complexes with each aryl boronic acid on its catechol moiety quantitatively to produce a fluorescence signal $\left(\lambda_{\text {ex }}\right.$ $\left.=495 \mathrm{~nm}, \lambda_{\mathrm{em}}=600 \mathrm{~nm}\right) .{ }^{[20]}$ As observed, the quenching in fluorescence upon the addition of dendron $\mathbf{5}$ corresponds to the displacement of Alizarin Red S from the protein surface (Figure 1a). The affinity constants of boronic acid/catechol and boronic acid/salicyl hydroxamate at $\mathrm{pH} 7.4$ were reported to be $800 \mathrm{M}^{-1}$ and $17,800 \mathrm{M}^{-1}$ respectively, ${ }^{[16,21]}$ allowing the quantification of the number of boronic acid groups and dendrons bound on each enzyme molecule through a fluorescence based titration. For each protein, the end point from the titration of dendron 5 (10 e.q. for trypsin and papain, 4 e.q. for DNase I) corresponds to the results obtained earlier by MALDI-TOF analysis.

To evaluate the protection efficiency of the dendritic assembly, the proteolytic activity of the boronic acid modified proteases (trypsin and papain) were tested and quantified using $N_{\alpha}$-benzoyl- $L$ arginine 4-nitroanilide hydrochloride as the substrate. The dendrons were first conjugated onto both proteins by incubating both components at $\mathrm{pH} 7.4$ prior to the analysis. Incidentally, the activity of the hybrid construct was reduced to $<10 \%$, suggesting that the catalytic site of the enzymes was sterically hindered by the attached dendrons (Figure 1b, c). Upon reduction to $\mathrm{pH} 5.0$, the dendrons dissociated and were removed by ultrafiltration and the activity of the enzymes was measured again at $\mathrm{pH}$ 7.4. The recovery of activity upon the dissociation of dendrons was calculated to be $>90 \%$, supporting the reversibility of the boronic acid/salicyl hydroxamate binding. To further substantiate this observation, the dendronized proteases were tested at $\mathrm{pH} 7.4$ against human serum albumin (HSA) as a protein substrate followed by an SDS-PAGE analysis. The addition of dendron $\mathbf{5}$ to both boronic acid modified proteases significantly blocked the proteolytic activity, with observable native albumin $(67 \mathrm{kDa})$ clearly seen (Figure 1e, f). The reversibility of the protective dendritic shell was reinforced by performing the proteolytic digestion after the removal of the dendrons at $\mathrm{pH} 5.0$ (Figure S3 in Supporting Information). Similarly, the activity of modified DNase I was tested against a 2,000 base pair plasmid (pUC19) in which the degradation of the supercoiled plasmid into its linear form was effectively blocked in the presence of dendron $\mathbf{5}$ at pH 7.4 and subsequently recovered at pH 5.0 (Figure 1d).

The bioorthogonality and possible aggregation behaviour of the dendritic assembly was investigated using fluorescence polarization. The interaction between the boronic acid modified protease with dendron $\mathbf{5}$ demonstrated an expected increase in size and displayed consistency in serum fortified DMEM (Figure S4 in Supporting Information). The absence of aggregation behaviour of these dendronized proteins ${ }^{[22]}$ and the specificity of the boronic acid/salicyl hydroxamate interaction agree with previous reports. ${ }^{[16]}$
In addition to conferring $\mathrm{pH}$ dependent "assemble and release" properties to the modified enzymes, these positively charged dendrons facilitate cellular uptake through electrostatic interactions with the negatively charged cell membrane. The uptake proceeds via clathrin-dependent endocytosis and intracellular release is driven by the proton sponge effect as described previously. ${ }^{[14,23]}$ Consequently, these modified proteases showed efficient dendron mediated uptake observed through confocal microscopy (Figure 2). As the dissociation of the dendritic shell directly relied on low intracellular $\mathrm{pH}$, localization of these constructs in acidic compartments was necessary for the release of the active protease. Co-localization studies using LysoTracker ${ }^{\circledR}$, which is fluorescent in acidic organelles, directly showed that the dendronized proteins were indeed located within these cellular compartments. In contrast, the non-dendronized proteins displayed minimal cellular uptake.

Facilitated cellular entry by the dendritic shell, these proteases could become active when the attached dendrons dissociate and directly causing rampant proteolytic degradation and cell death. Hence, upon addition of these dendronized proteases into A549 cells, the impact on cell viability and their potency was immediately clear after $3 \mathrm{~h}$ of incubation at $37{ }^{\circ} \mathrm{C}, 5 \% \mathrm{CO}_{2}$. As controls, the respective native proteases, covalently dendronized G2-HSA and dendron 5 were used for comparison (Figure 3). The non-cytotoxic behaviour of both covalently and non-covalently dendronized HSA at much higher concentrations implied that the observed toxicity of the dendronized proteases cannot be attributed to the dendrons themselves or the cationic dendron-protein hybrid structure. These observations suggested that the dendritic shell not only plays an important role in the intracellular transportation but also as a benign sterical moiety.

In conclusion, we have presented the synthesis of a sterically demanding dendritic supramolecular protecting group that is responsive to changes between $\mathrm{pH} 7.4$ and $\mathrm{pH} 5.0$ by utilizing a bioorthogonal ligation method based on boronic acid/salicyl hydroxamate complexation. The dendritic protecting group demonstrated the shielding of large binding sites of macromolecules in a highly reversible fashion. In addition, the formation of a dendritic PAMAM shell imparts the capability for efficient membrane translocation and localization into the acidic intracellular compartments. These attributes are of high significance since the assembly and disassembly of the dendritic shell occurs at physiological conditions, conferring stability at near neutral $\mathrm{pH}$ and release inside acidic cellular lysosomes. The resulting active proteases in the cell severely decreased the cell viability. In perspective, this integrated bioorthogonal dendritic assembly provides a unique design uncovering a contemporary dimension in creating highly efficient hybrid zymogens as smart protein therapeutics.

Received: ((will be filled in by the editorial staff))

Published online on ((will be filled in by the editorial staff))

\section{Keywords: Supramolecular chemistry · Enzymes · Dendrimers}

[1] P. Plainkum, S. M. Fuchs, S. Wiyakrutta, R. T. Raines, Nat. Struct. Mol. Biol. 2003, 10, 115.

[2] A. V. Karginov, F. Ding, P. Kota, N. V. Dokholyan, K. M. Hahn, Nat. Biotechnol. 2010, 28, 743.

[3] C. Lopez-Otin, L. M. Matrisian, Nat. Rev. Cancer 2007, 7, 800.

[4] E. M. Sletten, C. R. Bertozzi, Angew. Chem. Int. Ed. 2009, 48, 6974.

[5] Z. Ding, C. J. Long, Y. Hayashi, E. V. Bulmus, A. S. Hoffman, P. S. Stayton, Bioconjugate Chem. 1999, 10, 395. 
[6] P. De, M. Li, S. R. Gondi, B. S. Sumerlin, J. Am. Chem. Soc. 2008, $130,11288$.

[7] P. S. Stayton, T. Shimoboji, C. Long, A. Chilkoti, G. Ghen, J. M. Harris, A. S. Hoffman, Nature 1995, 378, 472.

[8] W. Gao, W. Liu, T. Christensen, M. R. Zalutsky, A. Chilkoti, Proc. Natl. Acad. Sci. USA 2010, 107, 16432.

[9] M. Yan, J. Du, Z. Gu, M. Liang, Y. Hu, W. Zhang, S. Priceman, L. Wu, Z. H. Zhou, Z. Liu, T. Segura, Y. Tang, Y. Lu, Nat. Nanotechnol. 2010, $5,48$.

[10] A. A. Bastian, A. Marcozzi, A. Herrmann, Nat. Chem. 2012, 4, 789.

[11] D. Astruc, E. Boisselier, C. Ornelas, Chem. Rev. 2010, 110, 1857.

[12] L. Albertazzi, B. Storti, L. Marchetti, F. Beltram, J. Am. Chem. Soc. 2010, 132, 18158.

[13] S. K. Hamilton, E. Harth, ACS Nano 2009, 3, 402.

[14] D. Y. W. Ng, J. Fahrer, Y. Wu, K. Eisele, S. L. Kuan, H. Barth, T. Weil, Adv. Healthcare. Mater. 2013, DOI: 10.1002/adhm.201200419.

[15] M. L. Stolowitz, C. Ahlem, K. A. Hughes, R. J. Kaiser, E. A. Kesicki, G. Li, K. P. Lund, S. M. Torkelson, J. P. Wiley, Bioconjugate Chem. 2001, 12, 229.
[16] S. B. Y. Shin, R. D. Almeida, G. Gerona-Navarro, C. Bracken, S. R. Jaffrey, Chem. Biol. 2010, 17, 1171.

[17] C. Q. Pan, J. S. Uumer, A. Herzka, R. A. Lazarus, Protein Sci. 1998, 7, 628.

[18] P. Carter, J. A. Wells, Nature 1988, 332, 564.

[19] H. A. Chapman, R. J. Riese, G.-P. Shi, Annи. Rev. Physiol. 1997, 59, 63.

[20] G. Springsteen, B. Wang, Chem. Commun. 2001, 1608.

[21] G. Springsteen, B. Wang, Tetrahedron 2002, 58, 5291.

[22] S. L. Kuan, B. Stöckle, J. Reichenwallner, D. Y. W. Ng, Y. Wu, M. Doroshenko, K. Koynov, D. Hinderberger, K. Müllen, T. Weil, Biomacromolecules 2013, 14, 367.

[23] L. Albertazzi, M. Serresi, A. Albanese, F. Beltram, Mol. Pharm. 2010, 7,680 .

a)

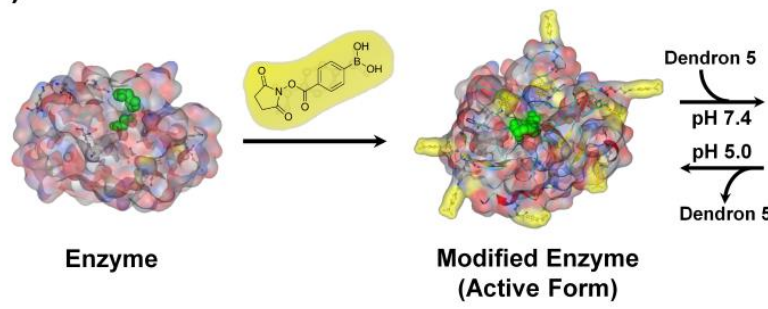

b)

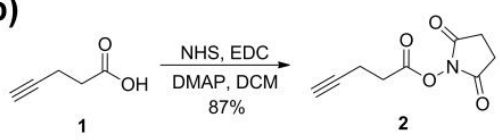
(Active Form)

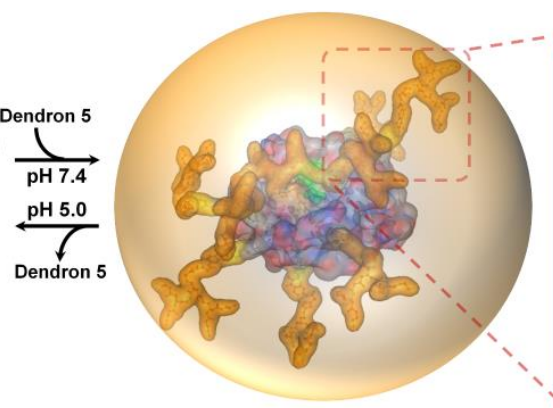

Protected "Zymogen" Form (Inactive)
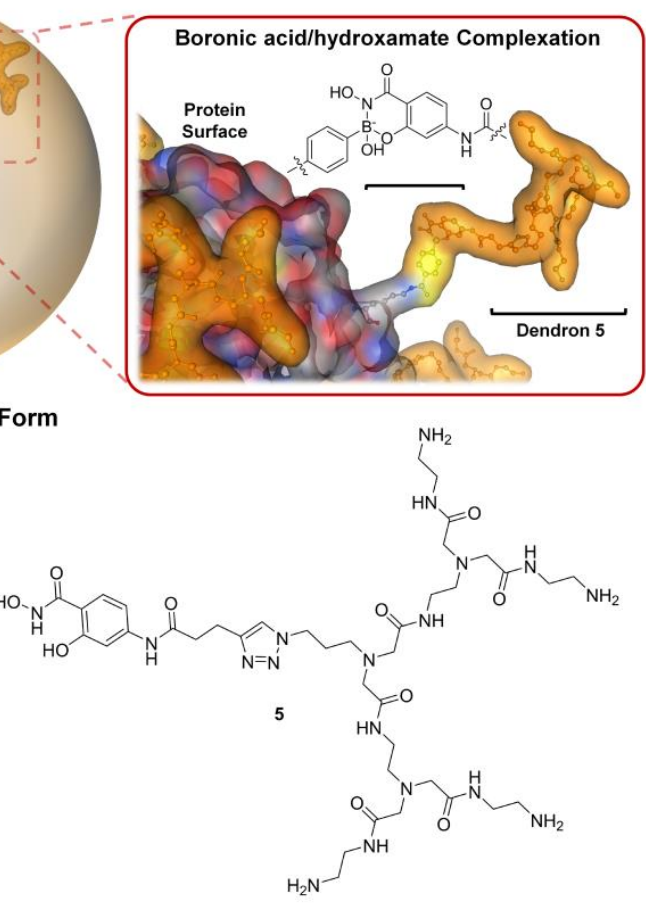

Scheme 1. a) Synthesis and supramolecular dendritic assembly for the construction of hybrid zymogens. Catalytic residues are highlighted in green. b) Synthesis of salicyl hydroxamate core dendron, (ii) 4-aminosalicylic acid. 
a)

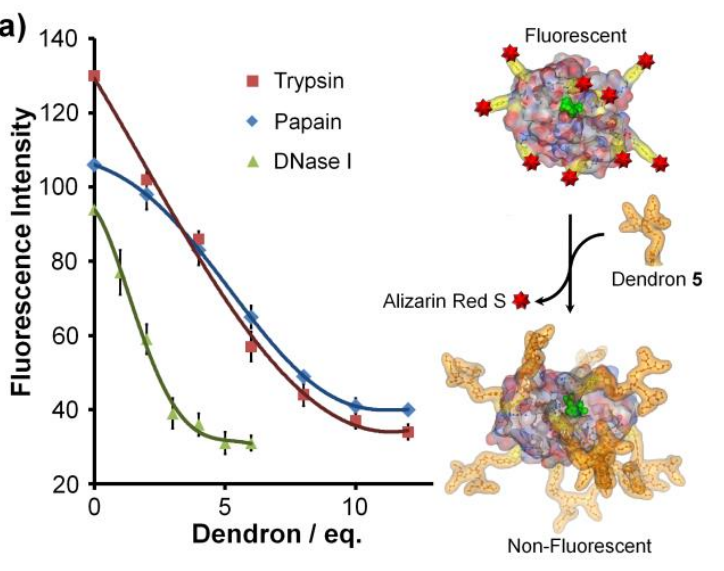

d)

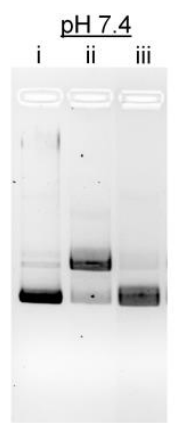

$\frac{\mathrm{PH} 5.0}{v}$

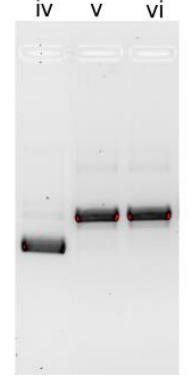

Plasmid ++++++

Modified $-++\infty++$

DNase 1

Dendron 5 b)

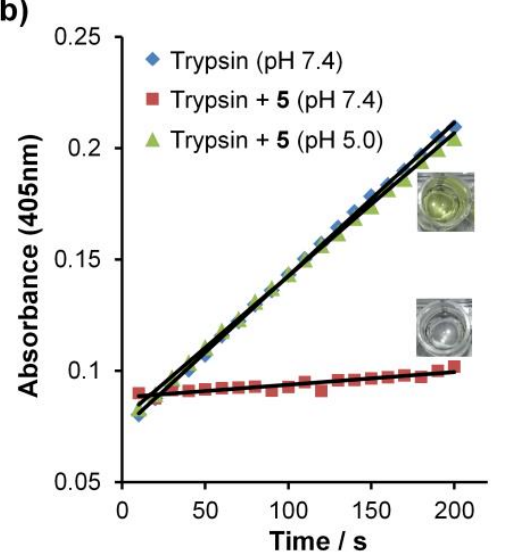

e)
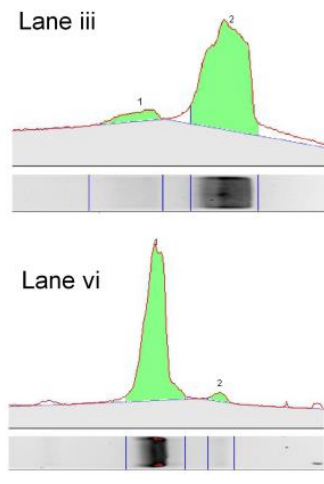

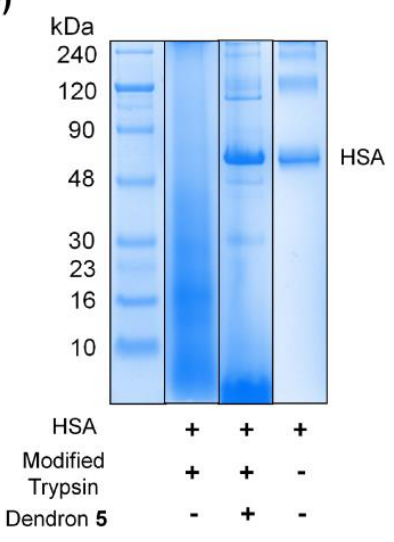

c)

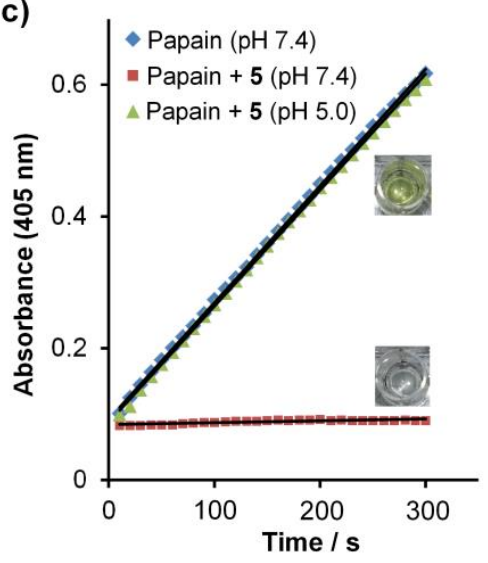

f)

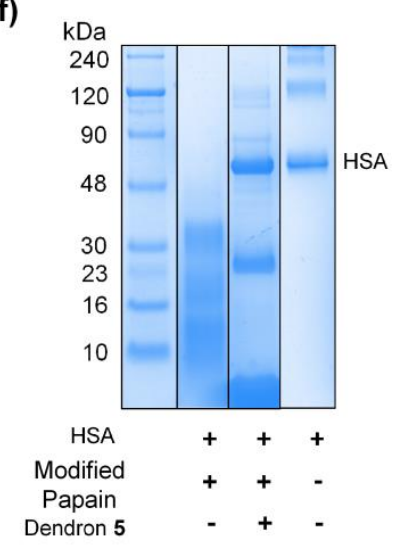

Figure 1. a) Fluorescence titration curves displaying the stoichiometric substitution of the fluorogenic Alizarin Red S/boronic acid complex on each protein by dendron 5 . b), c) Colorimetric enzyme kinetics for modified trypsin and papain based on $N_{\alpha}$-benzoyl- $L$-arginine 4-nitroanilide

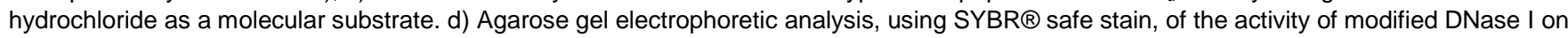
pUC19 DNA plasmid. Fluorescence intensity of bands in lanes iii and vi are shown in detail. e), f) SDS-PAGE analysis of the activity of modified trypsin and papain on human serum albumin as a protein substrate. 


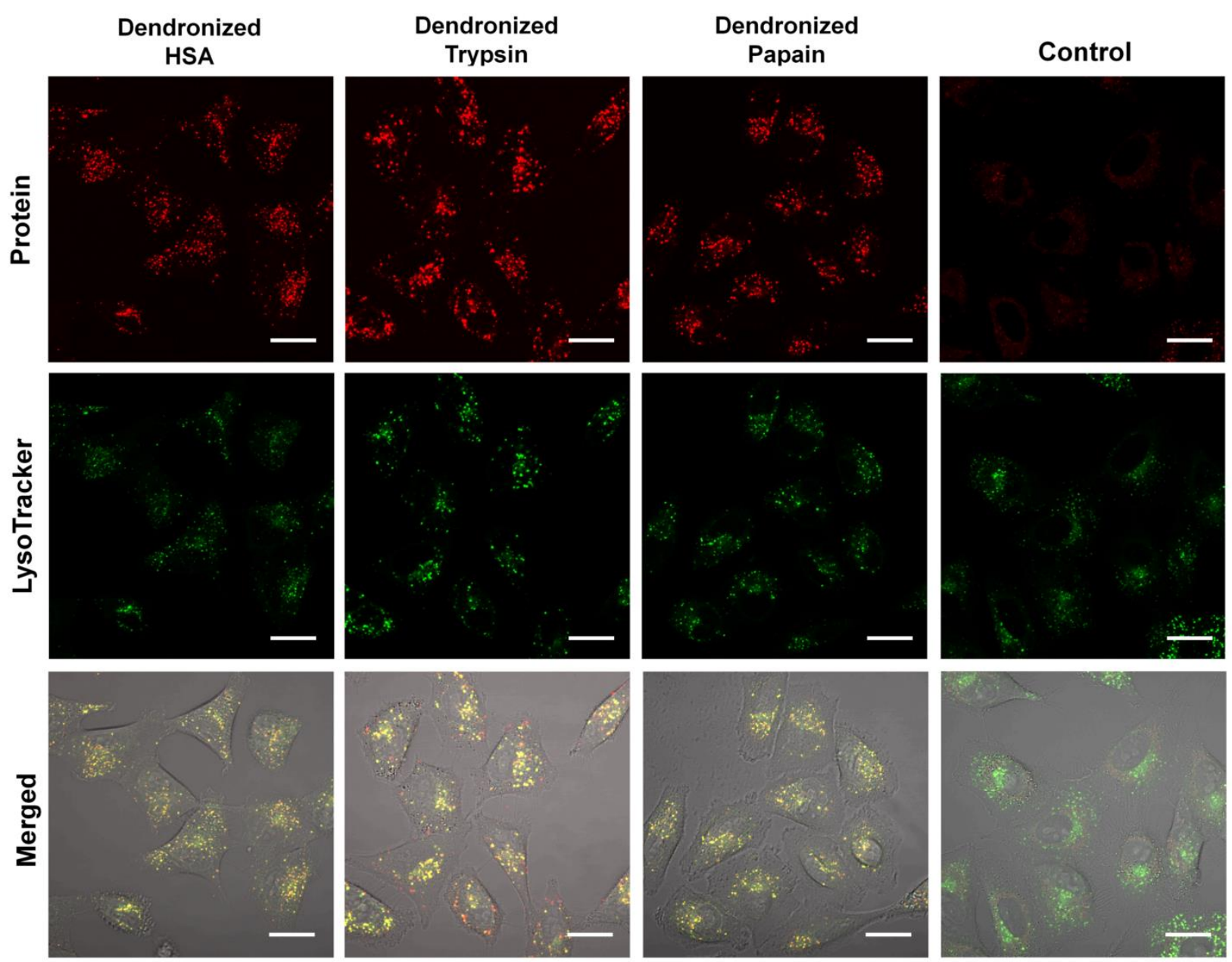

Figure 2. Confocal laser scanning microscopy using A549 cell lines. Co-localization of dendronized proteins (using Dylight 647 conjugated proteins) performed with LysoTracker ${ }^{\circledR}$. The control observation is representative of non-dendronized HSA, trypsin and papain. Scale bar: 20 $\mu \mathrm{m}$.

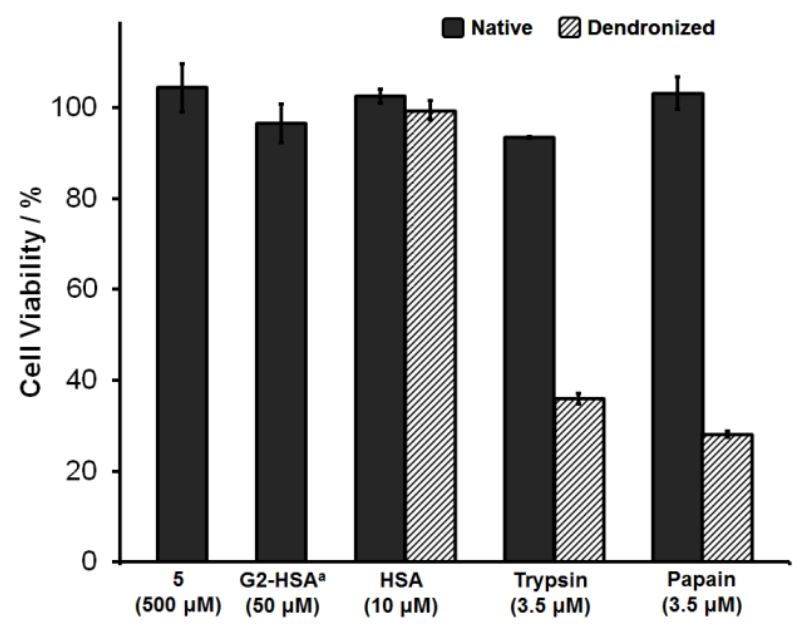

Figure 3. Cytotoxicity comparison between native proteases and their dendronized counterparts. Cell viability was referenced against blank controls. Data expressed as mean \pm SEM, $n=3$. ${ }^{a}$ G2-HSA was constructed by covalently conjugating 32 generation 2 PAMAM dendrons onto human serum albumin as previously reported. ${ }^{[22]}$ 
David Y.W. Ng, Matthias Arzt, Yuzhou Wu, Seah Ling Kuan, Markus Lamla and Tanja Weil* Page - Page

Constructing Hybrid Protein Zymogens through Protective Dendritic Assembly.

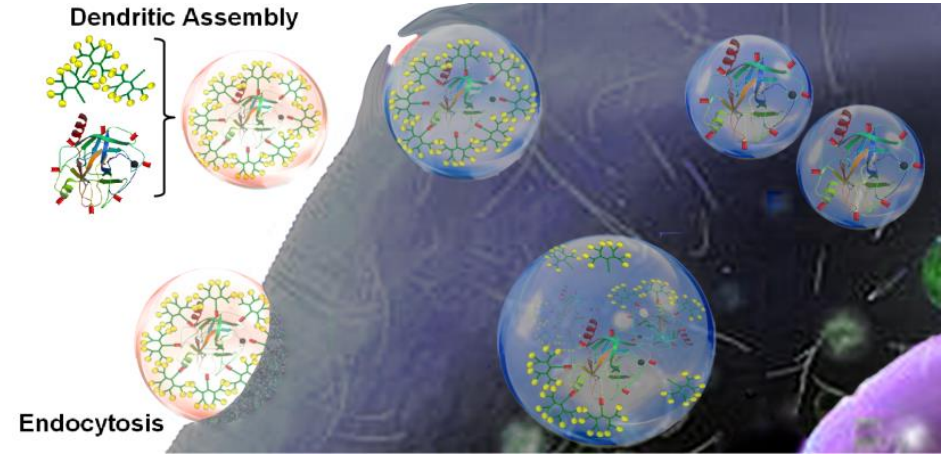

Programmed supramolecular chemistry was used to modulate enzyme activity by facile assembly of a dendritic shell onto enzymes as well as inducing cellular entry and release of the active proteins. The exclusively defined dendritic construct represents a contemporary avenue for smart protein therapeutics. 